\title{
ACCOUNTING FOR STATIC AND DYNAMIC OPEN WATER IN THE MODELING OF SMAP BRIGHTNESS TEMPERATURES OVER PEATLANDS
}

\author{
Bechtold, M. ${ }^{1,2}$, De Cannière, $S{ }^{1}$, Reichle. ${ }^{3}{ }^{3}$, De Lannoy, $G .{ }^{1}$ \\ ${ }^{1}$ KU Leuven, Dept Earth and Environmental Sciences, Belgium, \\ ${ }^{2}$ KU Leuven, Dept Computer Science, Belgium, \\ ${ }^{3}$ NASA Goddard Space Flight Center, Global Modeling and Assimilation Office, USA
}

\begin{abstract}
Hydrological change in peatlands due to anthropogenic disturbance and global warming can release enormous amounts of greenhouse gas emissions. Passive microwave satellite observations are an opportunity to globally monitor these changes. Abundant static and dynamic open water surfaces in peatlands strongly affect observed brightness temperatures (Tb). Here, we account for these contributions in radiative transfer modeling using NASA's Goddard Earth Observing System Model version 5 (GEOS-5) static open water mask and, for the dynamic open water fraction, the simulated inundated area using a version of the GEOS-5 Catchment land surface model that has been modified for peatland areas (PEAT-CLSM). Modeled Tb is compared against two years of SMAP L-band Tb. Preliminary results indicate: (i) a bias reduction when including the static open water fraction in a simple RTM mixing model, and ii) significantly improved correlation between modeled and observed $\mathrm{Tb}$ when using land surface output from PEAT-CLSM instead of the operational CLSM.
\end{abstract}

Index Terms - passive microwave, L-band, radiative transfer model, organic soil, soil moisture

\section{INTRODUCTION}

Peatlands represent a globally important carbon pool. About $500 \mathrm{Gt} \mathrm{C}$ is stored in peatlands of the Northern Hemisphere [1]. This enormous carbon pool is currently at risk. There are high peat carbon losses in regions of direct anthropogenic influence due to drainage for land use and forestry, and peat extraction for horticulture and energy use. More subtle disturbances of peatlands, but over much larger areas, occur by indirect anthropogenic influence, e.g. regional water level draw down due to groundwater extraction or global warming and changing precipitation dynamics [2].

Microwave remote sensing of soil moisture bears a high potential to provide valuable large-scale insights where and how the hydrological dynamics of peatlands is in transition

Thanks to the Alexander von Humboldt Foundation for funding. due to changing boundary conditions $[3,4]$. Being comparatively less affected by vegetation and more sensitive to moisture at greater soil depth (appr. 0-5 cm), the passive microwave missions SMOS and SMAP operating at L-band (1.4 $\mathrm{GHz}$ ) represent currently the most important technologies for large-scale observations of soil moisture. However, the differences between mineral and organic land surfaces have only been recently addressed in the scope of L-band radiometry, focusing on the specific dielectric properties of organic soils [5] and peatland vegetation [6], and on open water contributions to observed brightness temperatures [7].

Open water fractions within the footprint of L-band passive microwave observations have a strong impact on brightness temperature $(\mathrm{Tb})$ observations. Temporal changes in the extent of smaller water bodies as small as a few percent of the sensor footprint size are known to cause significant and dynamic biases [8]. Dynamical open water is difficult to model, and in case of peatlands, current model structures of global land surface models are not able to reproduce typical hydrological dynamics in peatlands well.

Here, we use output from the GEOS-5 Catchment Land Surface Model (CLSM) [9] in radiative transfer modeling (RTM) to simulate L-band Tb over peatlands with various amounts of static and dynamic open water fractions. We use SMAP $\mathrm{Tb}$ observations to evaluate the simulated $\mathrm{Tb}$ using two different CLSM versions and various configurations of mixing land and open water contributions.

\section{DATA}

Our study area is a set of 1500 EASEv2 $36 \mathrm{~km}$ grid cells over Siberia and Canada (Hudson Bay area) for which peat is the dominant soil type. Selected grid cells are south of the permafrost belt and have a relatively low tree cover of $<50 \%$. Over the selected peatlands, the static open water fraction ranges from 0 to about $50 \%$.

The EASEv2 $36 \mathrm{~km}$ SMAP Level 1C L-band Tb data (source: nsidc.org) were extracted from April 2015 to April 2017. Preliminary results of this abstract did only consider data in $\mathrm{H}$ polarization. 
Land surface state variables are simulated at the same EASEv2 $36 \mathrm{~km}$ resolution using (i) the operational CLSM of the SMAP Level 4 Soil Moisture product that takes advantage of a recently revised soil information including a peat class [10], and (ii) a new version of CLSM, hereafter called PEATCLSM, that uses a modified model structure over peat dominated grid cells. Major characteristics of peatland hydrology have been implemented as new modules into PEAT-CLSM:

- Surface Water Storage: water can pond in microrelief. Water table dependent total specific yield calculated as average of soil and open water specific yields [11]

- Single runoff function replacing original baseflow and overland flow functions $[12,13]$

- Evapotranspiration: water stress linked to water table depth

\section{METHODS}

Tb is estimated using different versions of RTM mixing models. The individual components are:

$T b_{\text {land }}$ : For non-inundated land, the L-band tau-omega RTM is used to estimate $36 \mathrm{~km} \mathrm{L-band} \mathrm{Tb}$ from $36 \mathrm{~km}$ CLSM simulated soil moisture and temperature. Calibrated RTM parameters from [14] were used. $36 \mathrm{~km}$ grid cells with open water fractions $>5 \%$ were masked in [14] and filled with globally averaged parameters for the same dominant vegetation class. $T b_{\text {land }}$ is simulated using either the operational CLSM or the modified PEAT-CLSM:

$$
\begin{aligned}
& T b_{L A 1}=T b_{\text {land,CLSM }} \\
& T b_{L A 2}=T b_{\text {land,PEAT-CLSM }}
\end{aligned}
$$

$T b_{\text {stat }}$ : Static open water surfaces are assumed to be vegetation-free. Assuming that the temperature of the water surface equals the CLSM simulated temperature of the topsoil, the dielectric permittivity of water and subsequently $T b_{\text {stat }}$ is calculated assuming a smooth water surface [15].

$T b_{d y n}$ : Regarding the dynamical fraction of the open water, we calculate two different $\mathrm{Tb}$ that we apply to different mixing model variants. Besides $T b_{\text {dyn, noveg }}$ which equals $T b_{\text {stat }}$, we also model $T b_{\text {dyn,veg }}$ using the tau-omega RTM assuming a smooth surface but using the same vegetation parameters.

These $\mathrm{Tb}$ contributions over static and dynamic open water are mixed with the $\mathrm{Tb}$ contribution over land in 3 distinct ways: :

$$
\begin{aligned}
T b_{O W 1}= & \left\{\left(1-f_{O W, \text { stat }}\right) \times T b_{L A 2}\right. \\
& \left.+\left(f_{O W, \text { stat }}\right) \times T b_{\text {stat }}\right\} \\
T b_{O W 2}= & \left\{\left(1-f_{O W, \text { stat }+ \text { dyn }}\right) \times T b_{L A 2}\right. \\
& \left.+\left(f_{O W, \text { stat }+ \text { dyn }}\right) \times T b_{\text {stat }}\right\}
\end{aligned}
$$

$$
\begin{aligned}
T b_{O W 3}= & \left\{\left(1-f_{O W, \text { stat }+ \text { dyn }}\right) \times T b_{L A 2}\right. \\
& +\left(f_{O W, \text { stat }}\right) \times T b_{\text {stat }} \\
& \left.+\left(f_{O W, \text { dyn }}\right) \times T b_{\text {dyn }, \text { veg }}\right\}
\end{aligned}
$$

Because PEAT-CLSM has not been calibrated, we assume that the absolute level of the water table depth, and consequently the fraction of dynamical inundation, can be strongly biased locally, overestimating or underestimating the open water fraction. Therefore, additionally $T b_{O W 3}^{*}$ was estimated using a pixel-specific factor to scale $f_{O W, d y n}$ so that the correlation coefficient between $T b_{O W 3}^{*}$ and $T b_{o b s}$ is maximal.

\section{PRELIMINARY RESULTS AND DISCUSSION}

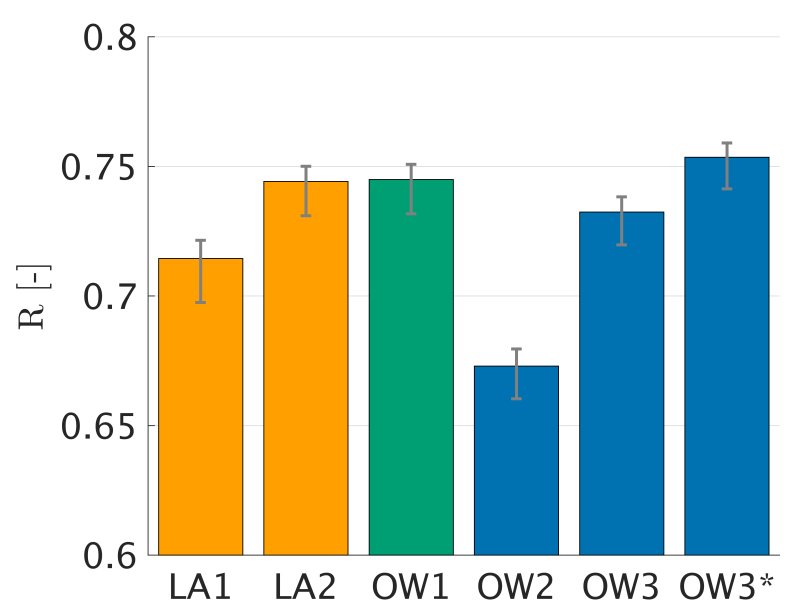

Fig. 1. Pearson correlation coefficient between SMAP Tb and modeled $\mathrm{Tb}$ for different scenarios. Mean and the $95 \%$ confidence intervals are obtained for a period of 2 years and after averaging across 1500 pixels with dominant peat soils.

Figure 1 shows the temporal correlation coefficients of the different $\mathrm{Tb}$ simulations. The currently operational CLSM does not simulate a dynamical open water fraction $T b_{L A 1}$. $T b_{L A 2}$ based on PEAT-CLSM is the equivalent land-alone estimate to $T b_{L A 1}$, i.e. without accounting for open water, even if it is simulated. $T b_{L A 2}$ performs significantly better than $T b_{L A 1}$ probably due to the more realistic representation of peatland hydrology in PEAT-CLSM. Including the static open water fraction $\left(T b_{O W 1}\right)$ does not further improve the correlation coefficient, but reduces bias related to lack of open water in $T b_{L A 2}$, as illustrated in Figure 2. The two estimates $T b_{O W 2}$ and $T b_{O W 3}$ include the modeled dynamical open water fraction in a mixing model approach, either without or with vegetation. The correlations does not improve, which may be due to RTM parameters which may partly compensate for open water. . However, it can be noted that $T b_{O W 3}$ results in a much higher $\mathrm{R}$ than $T b_{\mathrm{OW} 2}$. It seems thus more appropriate to assume a rather incomplete inundation for the 

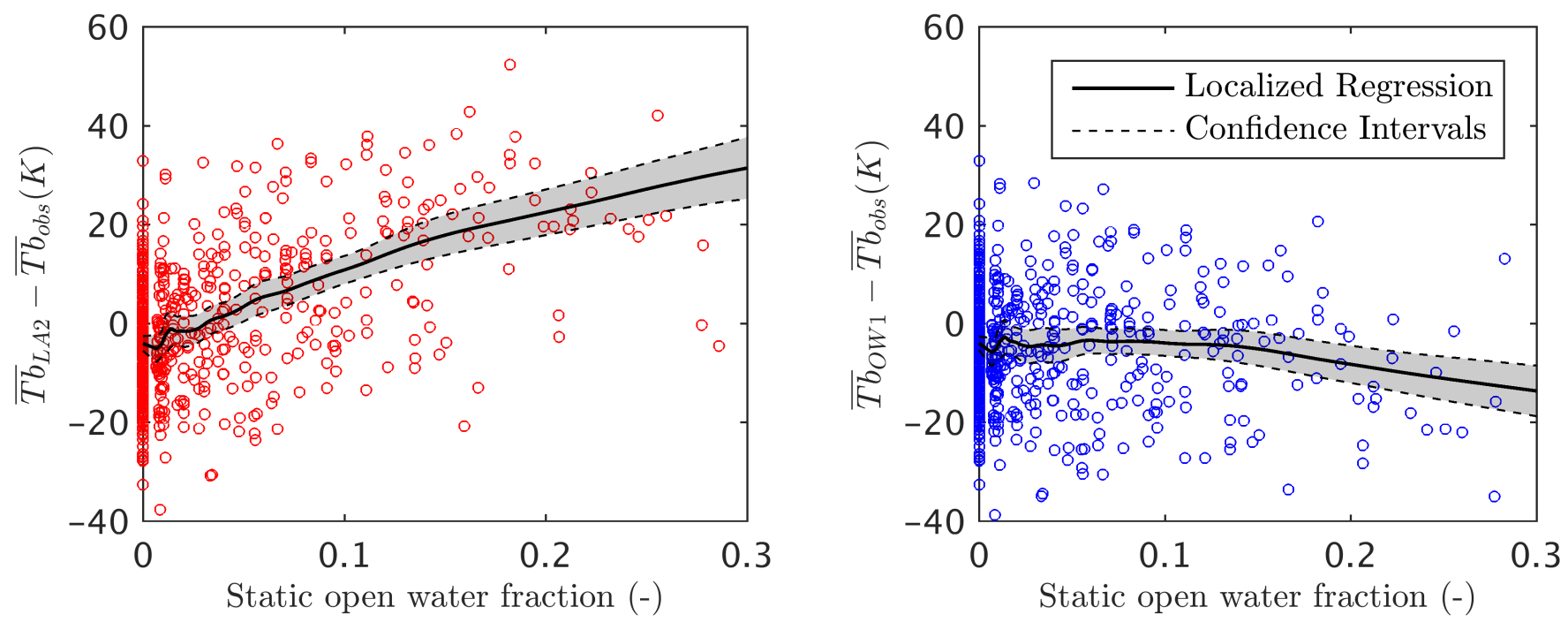

Fig. 2. Bias (model - observation) as a function of static open water fraction for the temporally averaged modeled brightness temperatures $T b_{L A 2}$ (neglecting open water) and $T b_{O W 1}$ (accounting for static open water.)

dynamical open water fraction in peatlands, i.e. significantly amount of the vegetation mass is still above the water surface. Finally, the first attempt of spatially fine-tuning PEATCLSM, by "post-scaling" the modeled dynamical fraction to obtain $T b_{O W 3}^{*}$, resulted tentatively, yet insignificant, into the highest $\mathrm{R}$. The preliminary results from the mixing model $\mathrm{Tb}$ estimates indicate the strong impact of including dynamical open water surfaces into $\mathrm{Tb}$ modeling. Biased modeled results for the open water fraction can have a strong negative effect on the estimated $\mathrm{Tb}$. This is in particularly the case when assuming that the dynamically inundated land is open water that is totally free of vegetation, which results in much lower brightness temperatures than open water surfaces with vegetation.

\section{OUTLOOK}

The current RTM parameters are probably suboptimal over peatlands, and a recalibration that already includes, at least, the static open water component in the forward modeling, should remove the bias with static open water fraction and lead to more consistent RTM parameters. We will conduct a recalibration for both CLSM and PEAT-CLSM output.

The applied scaling of the dynamically inundated area used for $T b_{O W 3}^{*}$ produces inconsistent hydrological variables, because the dynamics of the modeled soil moisture must change accordingly. We therefore run PEAT-CLSM for different absolute moisture levels, i.e. more specifically for a set of vertically-shifted runoff functions. This will yield a consistent set of soil moisture and partial inundation time series that can be applied to the set of RTM mixing models.

\section{REFERENCES}

[1] Z C Yu, "Northern peatland carbon stocks and dynamics: a review," Biogeosciences, vol. 9, no. 10, pp. 40714085, 2012.

[2] S Frolking, J Talbot, M C Jones, C C Treat, J B Kauffman, E S Tuittila, and N Roulet, "Peatlands in the Earth's 21 st century climate system," Environmental reviews, vol. 19, pp. 371-396, 2011.

[3] Jin Woo Kim, Zhong Lu, Laurel Gutenberg, and Zhiliang Zhu, "Characterizing hydrologic changes of the Great Dismal Swamp using SAR/InSAR," Remote Sensing of Environment, vol. 198, pp. 187-202, 2017.

[4] Michel Bechtold, Stefan Schlaffer, Bärbel Tiemeyer, and Gabriëlle J.M. De Lannoy, "Inferring Water Table Depth Dynamics from ENVISAT-ASAR C-Band Backscatter over a Range of Peatlands from DeeplyDrained to Natural Conditions," Remote Sensing, vol. 10, no. 4, 2018.

[5] Simone Bircher, François Demontoux, Stephen Razafindratsima, Elena Zakharova, Matthias Drusch, Jean Pierre Wigneron, and Yann H. Kerr, "L-band relative permittivity of organic soil surface layers-A new dataset of resonant cavity measurements and model evaluation," Remote Sensing, vol. 8, no. 12, 2016.

[6] Franois Jonard, Simone Bircher, François Demontoux, Lutz Weihermüller, Stephen Razafindratsima, Jean Pierre Wigneron, and Harry Vereecken, "Passive L-Band Microwave Remote Sensing of Organic Soil 
Surface Layers: A Tower-Based Experiment," Remote Sensing, vol. 10, no. 2, 2018.

[7] Seungbum Kim, Brian Brisco, and Valentin Poncos, "Inundation extent monitoring with smap data for carbon studies," in IEEE International Geoscience and Remote Sensing Symposium (IGARSS), Fort Worth, TX, USA, 2017.

[8] B. T. Gouweleeuw, A. I.J. M. Van Dijk, J. P. Guerschman, P. Dyce, and M. Owe, "Space-based passive microwave soil moisture retrievals and the correction for a dynamic open water fraction," Hydrology and Earth System Sciences, vol. 16, no. 6, pp. 1635-1645, 2012.

[9] Randal D. Koster, Max J. Suarez, Agnès Ducharne, Marc Stieglitz, and Praveen Kumar, "A catchmentbased approach to modeling land surface processes in a general circulation model: 1. Model structure," Journal of Geophysical Research, vol. 105, no. D20, pp. 24809, 2000.

[10] Gabriëlle J.M. De Lannoy, Randal D. Koster, Rolf H. Reichle, Sarith P.P. Mahanama, and Qing Liu, "An updated treatment of soil texture and associated hydraulic properties in a global land modeling system," Journal of Advances in Modeling Earth Systems, vol. 6, no. 4, pp. 957-979, 2015.

[11] U. Dettmann and M. Bechtold, "One-dimensional expression to calculate specific yield for shallow groundwater systems with microrelief," Hydrological Processes, vol. 30, no. 2, 2016.

[12] V. V. Romanov, Hydrophysics of bogs, Israel Program of Scientific Translations, Jerusalem, 1968.

[13] K.E. Ivanov, Water movement in Mirelands, Academic Press, London, 1981.

[14] Gabriëlle J.M. De Lannoy and Rolf H. Reichle, "Assimilation of SMOS brightness temperatures or soil moisture retrievals into a land surface model," Hydrology and Earth System Sciences, vol. 20, no. 12, pp. 48954911, 2016.

[15] W. T. Crow, S. Chant, D. Entekhabi, A. Hsu, T. J. Jackson, E. Njoku, P. O'Neill, and J. Shi, "An observing system simulation experiment for hydros radiometeronly soil moisture and freeze-thaw products," in International Geoscience and Remote Sensing Symposium (IGARSS), 2005, vol. 4, pp. 2737-2740. 